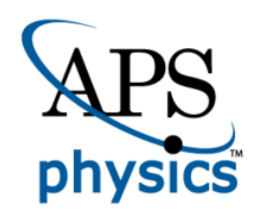

This is the accepted manuscript made available via CHORUS. The article has been published as:

\title{
Heterogeneities of flow in stochastically generated porous media
}

Jeffrey D. Hyman, Piotr K. Smolarkiewicz, and C. Larrabee Winter

Phys. Rev. E 86, 056701 - Published 1 November 2012 DOI: 10.1103/PhysRevE.86.056701 


\title{
Heterogeneities of Flow in Stochastically Generated Porous Media
}

\author{
Jeffrey D. Hyman* \\ University of Arizona, Program in Applied Mathematics, Tucson, AZ, 85721-0089, USA \\ Piotr K. Smolarkiewicz ${ }^{\dagger}$ \\ National Center for Atmospheric Research, Mesoscale and Microscale Meteorology, Boulder, CO, 80307-3000, USA \\ C. Larrabee Winter $\ddagger$ \\ University of Arizona, Department of Hydrology and Water Resources, \\ Program in Applied Mathematics, Tucson, AZ, 85721-0011, USA
}

\begin{abstract}
Heterogeneous flows are observed to result from variations in the geometry and topology of pore structures within stochastically generated three dimensional porous media. A stochastic procedure generates media comprised of complex networks of connected pores. Inside each pore space, the Navier-Stokes equations are numerically integrated until steady state velocity and pressure fields are attained. The intricate pore structures exert spatially variable resistance on the fluid, and resulting velocity fields have a wide range of magnitudes and directions. Spatially non-uniform fluid fluxes are observed, resulting in principal pathways of flow through the media. In some realizations, up to $25 \%$ of the flux occurs in $5 \%$ of the pore space depending on porosity. The degree of heterogeneity in the flow is quantified over a range of porosities by tracking particle trajectories and calculating their attributes including tortuosity, length, and first passage time. A representative elementary volume is first computed so the dependence of particle based attributes on the size of the domain through which they are followed is minimal. High correlations between the dimensionless quantities of porosity and tortuosity are calculated and a logarithmic relationship is proposed. As the porosity of a medium increases the flow field becomes more uniform.
\end{abstract}

PACS numbers: 47.56.+r, 47.15.G-, 47.11.Bc, 91.60.Np

Keywords: flow in porous media, first passage percolation, immersed boundary method, porosity, principal pathways, tortuosity

\section{INTRODUCTION}

Microscopic spatial variations of a porous medium manifest themselves through the geometry of the pore space (pore throat radii, the local curvature of pores) and its topology (degree of connectivity). These variations induce heterogeneities in the flow of a fluid moving through a porous medium. Resulting heterogeneities in the velocity field are reflected in the particle trajectories and bundles of trajectories defining principal pathways of flow. The ability to investigate details of flow within a pore network is important as it can guide applications ranging from design of engineered materials to evaluation of environmental risks. Detailed predictions of fluxes in pore networks can be limited by observational constraints and analytical inadequacies in dealing with complex boundary conditions arising from variable material properties [1-4]. Computational experiments are alternatives for investigating detailed characteristics of flow within networks of pores and can yield detailed velocity and pressure fields in complex pore spaces [5-19].

Multiple techniques are in use for numerically modeling flow through explicit pore spaces. Lagrangian parti-

\footnotetext{
*Corresponding author: jhyman@math.arizona.edu

† smolar@ucar.edu

$\ddagger_{\text {winter@email . arizona.edu }}$
}

cle methods such as lattice Boltzmann $[5,6,9,10]$ and smooth particle hydrodynamics $[11,12,15,17]$ have been used to resolve both single and multiphase flows in arbitrary pore geometries. Eulerian methods integrate the Navier-Stokes equations using finite volume or finite element techniques on meshes generated in explicit porespaces $[14,19]$. The immersed boundary method, the alternative used here, inserts fictitious body forces into the Navier-Stokes equations to represent the pore space [18].

This paper investigates the following aspects of flow in porous media: how porosity impacts variations in the velocity field; particle based attributes of tortuosity, trajectory length, and percolation time; and principal pathways, which are comprised of trajectory bundles. The methods for generating each porous medium, numerically integrating the Navier-Stokes equations, and tracking particles through the fluid velocity fields are discussed in Section II. Multiple independent realizations of pore spaces are stochastically generated for a range of porosities. Each realization is comprised of complex networks of connected pores with varying degrees of pore throat radii and connectivity (Section II A). Velocity and pressure fields are obtained for each realization using the immersed boundary method (Section IIB). Fluid particles advected with the steady state flow field are followed through every pore space realization using a particle tracker (Section II C).

The combination of the particle trajectory attributes (tortuosity, first passage time, and trajectory length) pro- 
vides a portrait of a particle's journey inside of a porous medium. These particle attributes vary considerably across pore spaces with different values of porosity (Section III A) and depend upon the extent of the domain through which particles are tracked (Section III B). The tortuosity of a particle's trajectory quantifies its deviation from the straight line between its end points (Section III C). The first passage percolation time of a particle trajectory relates to a particle's mean velocity (Section III D) while trajectory length is a primary integral property of a particle's motion (Section IIIE). Heterogeneity in a velocity field produces bundles of particle trajectories which form in areas of greater fluid flux, i.e. principal pathways (Section IV). We close with a discussion of the results (Section V).

\section{METHODS}

\section{A. Pore Space Construction}

Each virtual porous medium is a realization drawn from an ensemble of pore spaces in $\mathbb{R}^{3}$ with sides of length $L_{x}=L_{y}=1.27 \cdot 10^{-2} \mathrm{~m}$ and $L_{z}=2.55 \cdot 10^{-2} \mathrm{~m}$, and volume $V=4.11 \cdot 10^{-6} \mathrm{~m}^{3}$. Level set percolation $[20,21]$ is used to generate the porous media. Each node on a three dimensional regular grid is assigned an independent identically distributed random value sampled from a uniform distribution on the closed interval $[0,1]$. This random field is convolved with a symmetric Gaussian kernel to generate an isotropic correlated random topography. The convolution is achieved by transforming the field into frequency space, multiplying it by a Gaussian function, and then transforming it back into real space. The correlation length of the topography is determined by the full half width at maximum of the Gaussian kernel, which is fixed at 0.0235 for all porous media in this study. Because the topographies are based on sums of independent uniform random variables weighted by a Gaussian kernel with unit $\mathcal{L}^{2}$ norm, topography values are approximately normally distributed with a mean of $1 / 2$ and variance of $1 / 12[22]$.

A level threshold, $\gamma \in(0,1)$, is applied to the resulting topography to determine which nodes are in the void space and which are in the solid matrix. If the value at a node is greater than $\gamma$, then the node is placed in the solid matrix, nodes with values below $\gamma$ are placed in the void space. Intuitively, as $\gamma$ increases so does the amount of void space in the porous medium. The result of applying this technique is a statistically stationary pore space in the sense that the finite-dimensional probability distributions of pore space membership are invariant with respect to translation in space.

For a fixed level of $\gamma$, the stochastic construction results in the variability of physical characteristics of the pore spaces such as porosity $n$ (the ratio of void volume over bulk volume). To study the influence of the threshold parameter on the expected porosity of a real- ization $n_{e}$, eleven different levels of $\gamma$ are selected and one thousand independent identically distributed pore space realizations are generated at each level. Each sample set has finite variance and therefore the strong law of large numbers (ergodicity) can be invoked to define the mean porosity of these sample sets as the expected porosity for a given level of $\gamma$. The relationship between threshold parameter $\gamma$ and the physical quantity $n_{e}$ is described by the function

$$
n_{e}(\gamma)=3.6 \gamma-1.3
$$

At $\gamma=0.36, n_{e}=0$ and at $\gamma=0.64, n_{e}=1$. Outside of $\gamma \in(0.36,0.64)$, this method for generating porous media ceases to produce physically meaningful structures. The correlation coefficient between $\gamma$ and $n_{e}$ is 0.99 , which indicates a strong linear relationship between the two. For physical intuition, $n_{e}$, rather than $\gamma$, is used to classify pore spaces.

Horizontal cross sections for three realizations of pore spaces are shown in Fig. 1. Black indicates a node in the solid matrix and white a node in the void space.
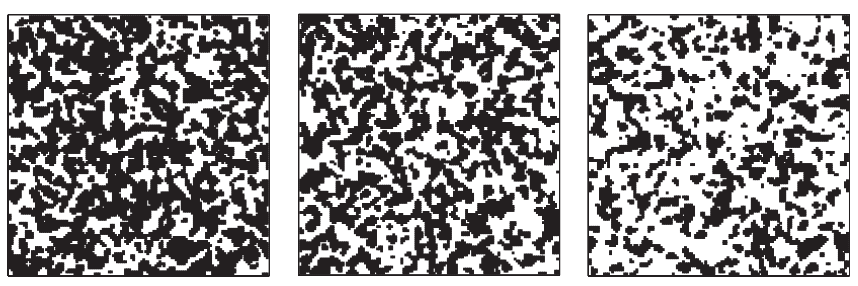

FIG. 1: Cross sections of pore spaces with $\gamma$ values and expected porosities of $(0.45,0.32),(0.50,0.50)$, and $(0.54,0.64)$, respectively. Black indicates a node in the solid matrix and white a node in the void space. As the threshold parameter, $\gamma$, increases the porosity increases.

\section{B. Computational fluid dynamics}

We follow the techniques of [18] for integrating the incompressible Navier-Stokes equations under gravity. Starting with a fluid at rest that fully saturates the pore space, the equations are integrated to attain steady state. One should refer to [18] for information regarding grid resolution, optimization of the numerics on larger clusters of processors, and a detailed description of the numerical methods used to integrate the governing equations although the method is sketched here for the reader's convenience.

Flow is simulated by numerically solving the incompressible Navier-Stokes equations on a Cartesian domain with a linear scale $\sim 10^{-2} \mathrm{~m}$. The grids for the virtual pore spaces have 128 nodes in the horizontal directions and 256 in the vertical direction. Each medium is periodic in the vertical direction with no flow allowed across lateral boundaries. The three components of velocity and 
pressure are computed at every point within each realization of a porous medium.

The multi-scale computational fluid dynamics modeling system EULAG [23] is used to numerically integrate the governing equations for water flow, as in [18]. The EULAG system accommodates a broad class of flows and underlying fluid equations in a variety of domains on scales ranging from wind tunnel and laboratory [24-26] through terrestrial environments and climate [27-30], to stellar [31].

The crux of our computational approach for simulating flows in porous media is an immersed-boundary method $[32,33]$ that inserts fictitious body forces in the equations of motion to mimic the presence of solid structures and internal boundaries. Conceptually, this is in the spirit of statistical theories that treat the solid phase as an external force field constraining the fluid to the void space $[34,35]$. The resulting dynamics are such that velocity is negligible and pressure irrelevant within the solid matrix where the body forces are high. The particular technique employed is a variant of feedback forcing [36], with implicit time discretization admitting rapid attenuation of the flow to stagnation within the solid matrix in $\mathcal{O}(\Delta t)$ time comparable to the time step $\Delta t=0.02 \mathrm{~s}$ of the fluid model. Flow simulations are run for 8 seconds with steady state conditions reached in 2-3 seconds.

For gravity-driven flows of a homogeneous incompressible fluid, e.g. water, through a porous medium, the Navier-Stokes equations are

$$
\begin{aligned}
\nabla \cdot \mathbf{v} & =0 \\
\frac{\partial \mathbf{v}}{\partial t}+\mathbf{v} \cdot \nabla \mathbf{v} & =-\nabla \pi^{\prime}+\mathbf{g}^{\prime}+\mu \triangle \mathbf{v}-\alpha \mathbf{v} .
\end{aligned}
$$

The primes refer to perturbations with respect to static ambient atmospheric conditions characterized by a constant density $\rho_{0}$ and pressure $p_{0}=p_{0}(z)$, so $\pi^{\prime}=$ $\left(p-p_{0}\right) / \rho$ and $\mathbf{g}^{\prime}=\left(0,0,-g \rho^{\prime} / \rho\right)$ where $\rho=$ const $\gg \rho_{0}$ denotes the density of fluid and $g$ is gravitational acceleration, $g=9.81 \mathrm{~m} / \mathrm{s}^{2}$. The kinematic viscosity of water $\mu$ is $10^{-6} \mathrm{~ms}^{-2}$.

The last term on the right hand side of the momentum equation is the fictitious repelling body force of the immersed boundary method, with a non-negative time scale $\alpha^{-1}(\mathbf{x})=0.5 \Delta t$ and the corresponding inverse time scale $\alpha(\mathbf{x})=0$ within the solid and fluid, respectively. Even though the mathematical form of the repelling body force $-\alpha \mathbf{v}$ is reminiscent of Stokes' drag, there is no physical connection between the two. The former is merely a mathematical prerequisite of the numerical device that circumvents the difficulty of imposing exact no-slip conditions along boundaries with complex geometry and topology. Intuitively, setting $\alpha(\mathbf{x})=0$ within the fluid admits Navier-Stokes flows away from the solid boundaries, while requiring $\alpha(\mathbf{x}) \rightarrow \infty$ within the solid assures $\mathbf{v} \rightarrow 0$ there.

Our implementation of the immersed boundary method is simple and effective but its application stands upon the strengths of the EULAG system. Since all calculations use exclusively the Eulerian option in a Cartesian-framework, the prognostic equations (2) can be idealized as

$$
\frac{\partial \psi}{\partial t}+\nabla \cdot(\mathbf{v} \psi)=F
$$

where $\psi$ symbolizes the components of $\mathbf{v}$ and $F$ stands for the corresponding right hand side. This system of the conservation laws is numerically integrated using a second-order-accurate, semi-implicit, non-oscillatory forward-in-time approach, whose theory, implementation and applications are broadly documented [37-39]. The resulting system is implicit with respect to pressure and all velocity components as all principal forcing terms are assumed to be unknown at the next time step. Its solution leads to an elliptic problem that is solved using a preconditioned non-symmetric Krylov-subspace solver $[40,41]$.

The nonlinear transport algorithm employed in the discretization of (3) suppresses spurious oscillations at sharp gradients of advected fields in the vicinity of the fluidsolid interface. This algorithm improves the conditioning of the explicit part of the elliptic problem (and thus the solver's convergence) and facilitates simulations with substantially larger Reynolds number than for creepingflow motions [18].

The advantage of this approach is its computational efficiency. One simulation with $\sim 4.2 \cdot 10^{6}$ degrees of freedom performed on a $\mathrm{PC}$ with a $3.2 \mathrm{GHz}$ processor completes in $\sim 4$ hours of CPU time compared to the $\sim 100$ hours of CPU time on a PC with a $3.0 \mathrm{GHz}$ processor required for the three dimensional smooth particle hydrodynamics simulation in [15] which consisted of a system with 72,169 particles. The method also bypasses strenuous unstructured mesh generation required in the approaches implemented in [14] and [19] as the mesh is a uniform Cartesian grid. The efficiency of this approach allows for many simulations to be preformed at a low cost and is especially appealing for Monte Carlo based experiments that require large numbers of stochastically generated pore space realizations.

This simple approach comes at a price. Unlike other immersed boundary methods, the pore space boundaries are aligned with the grid nodes and the resulting media are simulated only with first-order accuracy in space. However, the macroscopic uncertainty of microscopic pore structure greatly exceeds numerical inaccuracies in detailed representation of internal boundaries and therefore the first order approximation of a porous medium is adequate; cf. [42] for a quantitative substantiation and further discussion.

\section{Particle Tracking}

Every node in the void space at the top horizontal cross section of the steady state velocity field generated by EULAG is used as an initial position for a particle. Hence, 
the number of particles inserted into each realization depends upon its porosity. A fourth order Runge-Kutta scheme numerically integrates the trajectory equation,

$$
\frac{d \mathbf{x}\left(t, \mathbf{x}_{0}\right)}{d t}=\mathbf{v}\left(\mathbf{x}\left(t, \mathbf{x}_{0}\right)\right)
$$

of a particle with initial position $\mathbf{x}_{0}$. Trilinear interpolation is used to compute the particle velocity at locations away from computational nodes. Information about the initial location, current location, current trajectory length, travel time, minimum, and maximum velocity is recorded for each particle. Particles inserted in isolated pores (a pore that does not connect through the entire domain), do not move when their equation of motion is integrated. Conversely, except for the singular points in the flow (a topological set of measure zero), the particle that does not move must be in an isolated pore, therefore this is also the criterion for the detection. Conversely, except for the singular points in the flow (a topological set of measure zero), the particles that do not move must be in an isolated pore, therefore this is also the criterion for the detection.Since such particles do not percolate, they are identified and removed from further analysis.

Throughout this paper the tortuosity $\tau(a, b)=l_{s} / l$ of a trajectory connecting two points $a$ and $b$ is defined as the ratio of the total trajectory length $l_{s}$ over the Euclidean distance $l$ between $a$ and $b$. This definition of tortuosity results in values ranging between unity and infinity, $1 \leq$ $\tau(a, b)<\infty$. A number of alternate definitions are in use including $\tau^{2}, \tau^{-1}$, and $\tau^{-2}$ [43]. The mean tortuosity for particles advected through a prescribed domain,

$$
\bar{\tau}=\frac{1}{N} \sum_{i=1}^{N} \tau\left(a_{i}, b_{i}\right),
$$

is taken over $i=1, \ldots, N$ tortuosities $\tau\left(a_{i}, b_{i}\right)$ where $N$ is the number of particles that percolate. Other methods for calculating tortuosity are in use and a discussion of their benefits and drawbacks can be found in [44].

\section{PARTICLE TRAJECTORY ATTRIBUTES}

\section{A. Data Generation}

For the computational simulations of fluid flow, thirtysix new independent pore space realizations were generated with expected porosities $n_{e}$ derived in (1), with values ranging from 0.28 to 0.68 , within a normal range of actual porosity $n$ of $0.2<n<0.7$ as defined in [45]. These thirty-six were not among those used to define expected porosity $n_{e}$ in Section II A even though they are statistically identical. Three unique pore space realizations were created at twelve selected values of $n_{e}$. Of the thirty-six pore space realizations, thirty-four have actual porosities within the normal range and are included in the study. The combined sum of particles that percolate through all thirty-four pore space realizations is 38,146 . The number of particles that percolate through a single porous medium ranges from four to $4,008,(0.10 \%$ and $35.67 \%$ of the initial number of particles inserted, respectively).

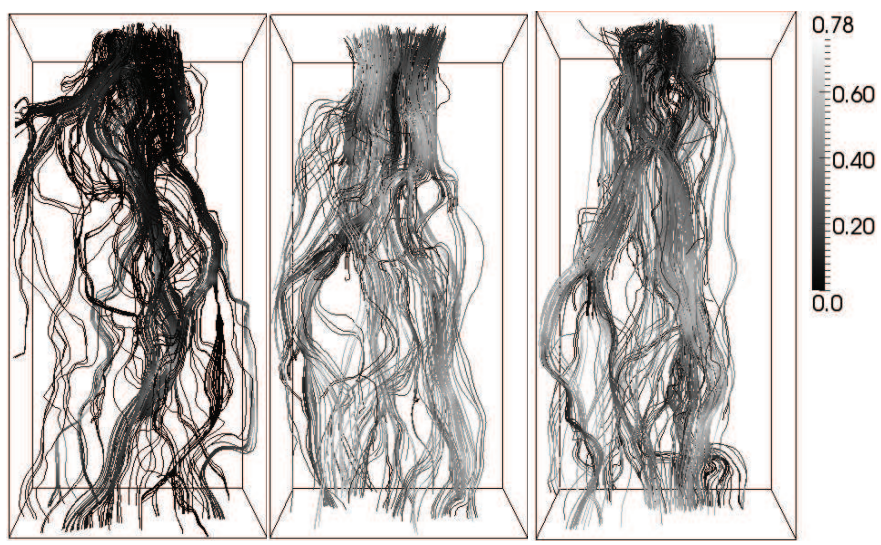

FIG. 2: Particle trajectories shaded by their normalized velocity magnitude $v_{0}=|\mathbf{v}| / \max |\mathbf{v}|$ within sub-volumes of pore space realizations with expected porosities of $0.32,0.50$, and 0.61 (left to right). The particle are inserted within a disk centered atop the domain with a radius $1 / 5$ the domain width and the direction of flow is from top to bottom. As $n_{e}$ increases the trajectories become straighter and more particles percolate through the domain. Additionally, there are more trajectories with higher normalized velocities.

Images of particle trajectories shaded according to their normalized velocities $v_{0}=|\mathbf{v}| / \max |\mathbf{v}|$, where $|\mathbf{v}|$ is the length of vector $\mathbf{v}$ and $\max |\mathbf{v}|$ is taken over the entire domain, are generated using the stream-tracer module in Paraview [46] and are displayed in Fig. 2. Each subfigure shows trajectories in a $1 / 64$ sub-volume of the entire domain, with linear dimensions $L_{x}=L_{y}=3.2 \cdot 10^{-3} \mathrm{~m}$ and $L_{z}=6.4 \cdot 10^{-3} \mathrm{~m}$. The entire domains have expected porosities of $0.32,0.50$, and 0.61 . For image clarity, the trajectories of only five hundred particles in each realization are shown. The particle are inserted within a disk centered atop the domain with a radius $1 / 5$ the domain width. In all of the subfigures, there are regions in the flow field where trajectories group together (bundle) and display higher velocities than the surrounding flow field. At the lowest value of $n_{e}$, the trajectories span a wide range of behaviors. As $n_{e}$ increases the trajectories straighten, have higher velocities, and more particles percolate. At the highest values of $n_{e}$ the trajectories are more uniform.

\section{B. Representative Elementary Volume}

A representative elementary volume (REV) of a porous medium is the smallest volume for which large fluctuations of observed quantities no longer occur [43]. To select an appropriate REV for particle based attributes, 
sub-volumes of the entire porous medium are sampled and particle tortuosities, trajectory lengths, and first passage times are measured and inspected to determine uniform ranges of small variation.

In [16], the authors used a lattice Boltzmann approach to simulate flow through two dimensional porous media composed of randomly placed squares and observed that the measured value of tortuosity depends upon the extent of the observation domain (a subsection of the entire domain through which particles are followed). Using different computational techniques, we extend their study to three dimensions and investigate the influence of observational domain extent on tortuosity as well as the dispersion of trajectory lengths and first passage time distributions.

Mean tortuosity (5) is calculated in observation domains extracted from three pore space realizations with expected porosities of $0.32,0.50$ and 0.68 . The horizontal area is fixed at $L_{x} \times L_{y}$ and the height of the observation domain $L$ is increased from $L=10 \cdot \Delta z$, to $L=250 \cdot \Delta z$ in intervals of $\Delta L=10 \cdot \Delta z$, the full domain, $L_{z}=255 \cdot \Delta z$, is also included. Free parameters $\left(\tau_{\infty}, a, b\right)$ in models of the form

$$
\mathcal{T}(L)=\tau_{\infty}-\exp (a-b L)
$$

are uniquely fitted for each pore space using the nonlinear fit module in Mathematica [47] and are shown in Table I along with the computed mean tortuosity for the entire domain. The computed mean tortuosity value from each sample observation domain is plotted in Fig. 3 along with the model (6) for each pore space.

TABLE I: Observation Domain Extent Fitting Parameters

\begin{tabular}{ccccc}
$n_{e}{ }^{a} \tau_{\infty}{ }^{b}$ & $a^{c}$ & $b^{d}$ & $\bar{\tau}\left(L_{z}\right)^{e}$ \\
\hline \hline 0.32 & 1.27 & -1.32 & 0.057 & 1.27 \\
0.50 & 1.16 & -2.08 & 0.054 & 1.16 \\
0.68 & 1.09 & -2.73 & 0.049 & 1.09 \\
\hline
\end{tabular}

${ }^{a}$ Expected porosity

${ }^{b}$ Asymptotic tortuosity

${ }^{c}$ First fitting parameter

${ }^{d}$ Second fitting parameter

${ }^{e}$ Computed mean tortuosity (5) for entire domain

At the lower values of observation domain extent, the computed mean tortuosity underestimates the tortuosity of the entire domain by an appreciable amount, and this underestimation is more significant for pore spaces with lower expected porosities. Although oscillations about the model (6) occur, Fig. 3 indicates that the computed mean tortuosity converges to $\tau_{\infty}$ exponentially with increasing observation domain extent. When the observation domain is the entire porous medium, the difference between $\bar{\tau}\left(L_{z}\right)$ and $\tau_{\infty}$ is minor, ranging between $6.6 \cdot 10^{-3}$ and $1.7 \cdot 10^{-5}$.

A similar study of the influence of observation domain extent on the coefficient of variation (the ratio of the standard deviation to the mean) computed for first passage

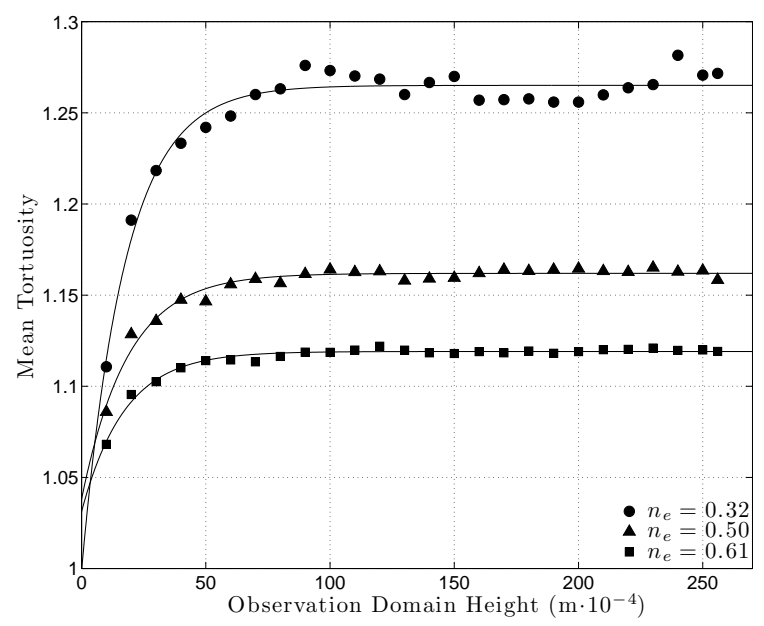

FIG. 3: Fitted models of the form $\mathcal{T}(L)=\tau_{\infty}-\exp (a-b L)$ are plotted along with the computed mean tortuosity (5) for pore spaces with three different expected porosities as a function of observation domain extent. As the height of the observational domain increases the mean tortuosity values converge to $\tau_{\infty}$. Stabilization occurs more rapidly in pore spaces with higher expected porosities.

time distributions and trajectory lengths reveals corresponding behavior. For lower observation domain extents, the coefficient of variation overestimates the value computed using the entire porous medium. As the extent of the observation domain increases the dependence on the height decays exponentially.

In order to minimize the dependence of measured particle based attributes on the extent of the observation domain, the entire porous medium is used as the observation domain for the primary investigation of how porosity influences tortuosities, trajectory lengths, and first passage time distributions of particle trajectories.

\section{Tortuosity and Porosity}

A relationship between porosity and tortuosity has been speculated by various researchers [5, 16, 48-53]. A single parameter model,

$$
\tau_{e}(n)=1-a \log (n),
$$

fits the data set well with $a=0.24$. The model is consistent with other models proposed in the literature $[16,50]$ and meets physical requirements; that is, if $n=1$, then $\tau_{e}=1$. Other linear and nonlinear models were also fitted to the data, but generated poorer matches and are not shown. For the data set, the correlation coefficient between expected porosity (1) and mean tortuosity (5) is -0.95 , and the correlation coefficient between actual porosity $n$ and mean tortuosity is -0.98 . 


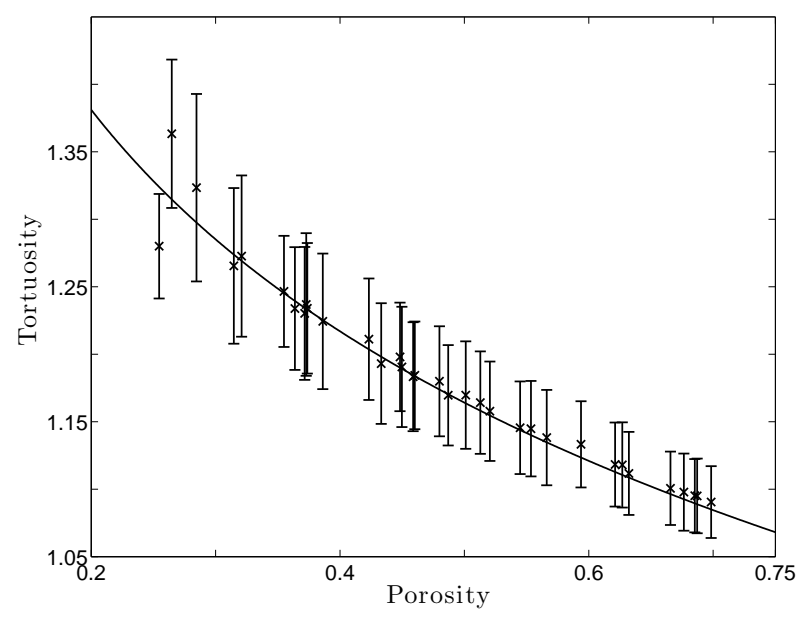

FIG. 4: Expected tortuosity as a function of porosity and the proposed model $\tau_{e}(n)=1-0.24 \log (n)$. Crosses indicate mean tortuosity for a realization and bars indicate one standard deviation.

Mean tortuosity along with the standard deviation of the entire set of particles that percolate through each pore space is plotted as a function of porosity along with the proposed fitted model (7) in Fig. 4. Crosses indicate mean tortuosity for a realization and error bars indicate one standard deviation. The variance of tortuosities decreases as $n$ increases and the model follows the nonlinear trend of $\bar{\tau}$.

\section{First Passage Time Distribution}

The first passage time distribution (FPTD) is the empirical distribution function generated by the time taken for particles to percolate all the way through a prescribed domain. These FPTDs are similar to the classically defined breakthrough curves attained via the standard continuum-scale advection dispersion equation. Each line in Fig. 5 is an FPTD generated using all percolating particles for a fixed level of expected porosity $n_{e}$. The highest value of $n_{e}, 0.68$, is on the left and the lowest value, 0.28 , is on the right. The number of particles that percolate for each set of pore space realizations of a particular value of $n_{e}$ ranges from $16(0.28)$ to $10,052(0.68)$. The sharpening of the empirical distribution functions indicates decreasing variance with increasing expected porosity. Also, the percent of particles with relatively large travel times decreases with higher expected porosity.

\section{E. Trajectory Lengths}

Six porous media are selected with expected porosity, $n_{e}$, values of $0.32,0.39,0.46,0.54,0.61$ and 0.68 and

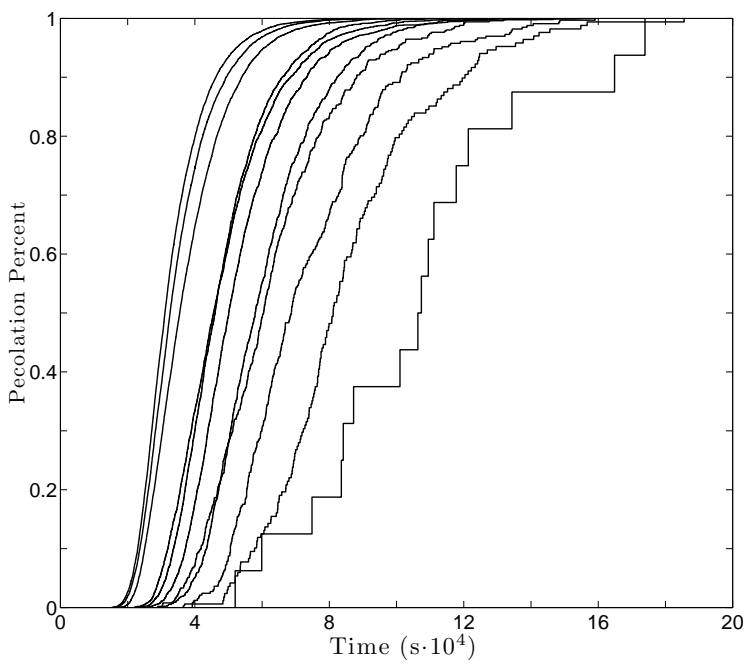

FIG. 5: FPTD: Lines correspond to first passage time distributions generated by particles percolating through the domain at a given time. Each line is generated using all percolating particles from the pore space realizations at a fixed value of expected porosity. From left to right lines correspond to realizations with $n_{e}$ values of $n_{e}=0.68$ decreasing to $n_{e}=0.28$.

a histogram of the particle trajectory lengths $l_{s}$ for particles that percolate through each pore space is shown in Fig. 6. The vertical length of the observation domain is $2.55 \cdot 10^{-2} \mathrm{~m}$. Out of all the particles trajectories, the minimum length is $2.61 \cdot 10^{-2} \mathrm{~m}$ and the maximum is $3.66 \cdot 10^{-2} \mathrm{~m}$. The mean, variance, minimum length, maximum length, skewness, and kurtosis for the trajectory lengths shown in Fig. 6 are displayed in Table II and discussed in Section V.

TABLE II: Distribution of trajectory lengths shown in Fig. 6

\begin{tabular}{rcccrrrc}
$n_{e}{ }^{a}$ Fig. $6^{b}$ & $\mu^{c}$ & $\sigma^{2}{ }^{d}$ & $\min \left(l_{s}\right)^{e}$ & $\max \left(l_{s}\right)^{f}$ & $s^{g}$ & $k^{h}$ \\
\hline \hline 0.32 & $\mathrm{a}$ & 3.261 & 0.025 & 3.023 & 3.610 & 0.227 & 1.929 \\
0.39 & $\mathrm{~b}$ & 3.169 & 0.019 & 2.883 & 3.536 & 0.500 & 2.741 \\
0.46 & $\mathrm{c}$ & 3.028 & 0.011 & 2.797 & 3.331 & 0.459 & 3.024 \\
0.54 & $\mathrm{~d}$ & 2.911 & 0.008 & 2.689 & 3.264 & 0.651 & 3.646 \\
0.61 & $\mathrm{e}$ & 2.859 & 0.007 & 2.669 & 3.204 & 0.677 & 3.891 \\
0.68 & $\mathrm{f}$ & 2.787 & 0.005 & 2.611 & 3.217 & 0.684 & 3.991 \\
\hline
\end{tabular}

${ }^{a}$ Expected porosity

${ }^{b}$ Fig. 6 subfigure

${ }^{c}$ Mean $\left[10^{-2} \mathrm{~m}\right]$

${ }^{d}$ Variance $\left[10^{-2} \mathrm{~m}\right]$

${ }^{e}$ Minimum trajectory length $\left[10^{-2} \mathrm{~m}\right]$

${ }^{f}$ Maximum trajectory length $\left[10^{-2} \mathrm{~m}\right]$

${ }^{g}$ Skewness

${ }^{h}$ Kurtosis 

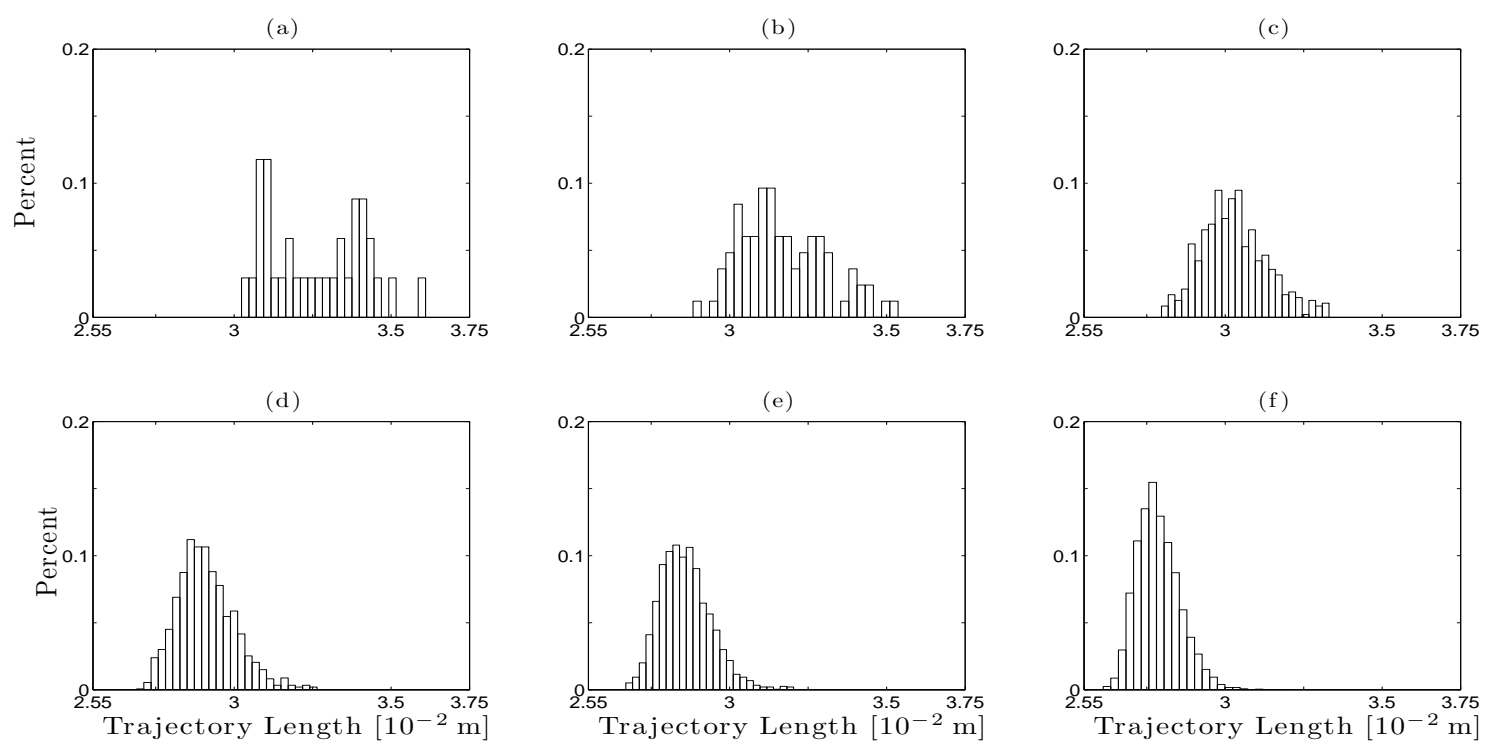

FIG. 6: Histogram of trajectory lengths arranged by expected porosities. (a) 0.32 , (b) 0.39 , (c) 0.46 , (d) 0.54 , (e) 0.61 and (f) 0.68. The straight line path is $L_{z}=2.55 \cdot 10^{-2} \mathrm{~m}$.

\section{PRINCIPAL PATHWAYS}

Since the fictitious internal boundaries are effectively established at steady state, the total mass flux out of every horizontal cross section must then be constant to satisfy mass continuity. Nonetheless, the velocity field within each cross section is nonuniform. This nonuniformity can result in principal pathways, large connected regions of high fluid velocity, through the pore space.

Normalized velocity magnitudes $v_{0}=|\mathbf{v}| / \max |\mathbf{v}|$ are calculated at horizontal cross sections within three pore space realizations with expected porosities, $n_{e}$, of $0.32,0.50$ and 0.61 . Ten uniform ranges of $v_{0}$ are selected and the percent of the void space area occupied by computational nodes with velocities in each range, as well as the percent of the total flux through the cross section transmitted at these nodes, is computed (Table III). At the lowest value of $n_{e}$ the distribution of both flux and area is highly nonuniform, e.g. $\sim 25 \%$ of the flux occurs in $\sim 5 \%$ of the area. The velocity field becomes more evenly distributed as the expected porosity increases.

Fig. 7 is a contour plot of $v_{0}$ in one-fourth of a horizontal cross section from the pore space realization with $n_{e}=0.61$ used in Table III. Some of the highest velocities occur in narrow pores, yet wider pores with high velocities also exist. Observations of particle exit locations reveals that they group together in areas of high velocity, indicative of particle trajectory bundles.

Images of 1,250 particle trajectories shaded according to $v_{0}$ in an observation domain extracted from a pore space realization with $n_{e}=0.61$ are generated using Paraview (Fig. 8). The sub-volume is $1 / 512$ the size

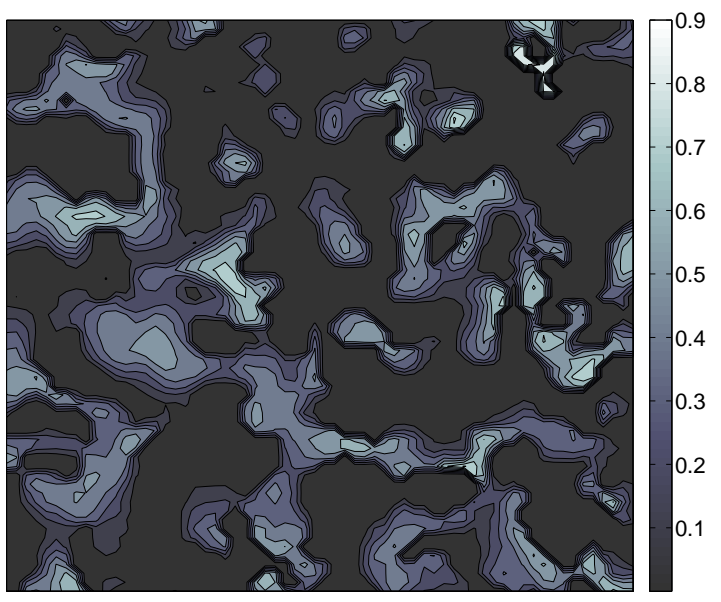

FIG. 7: Normalized velocity magnitude $v_{0}=|\mathbf{v}| / \max |\mathbf{v}|$ of the steady state solution to the Navier-Stokes equations in one-fourth of the bottom horizontal cross section extracted from a pore space with expected porosity of $n_{e}=0.61$.

of the entire domain, $L_{x}=L_{y}=1.6 \cdot 10^{-3} \mathrm{~m}$ and $L_{z}=3.2 \cdot 10^{-3} \mathrm{~m}$. The first image is rotated about the vertical axis 90 and 180 degrees in the second and third images to exhibit the three dimensional structure of the fluid flow. Since the sub-volume was taken out of the entire domain, some trajectories exit through the lateral boundaries. There are principal pathways where a large volume of fluid passes through connected regions of pores and also subsidiary pathways where only minor flow oc- 
TABLE III: Precent of total flux and percent of void space area for a horizontal cross section of pore space realizations with expected porosities of $n_{e}=0.32,0.50$ and 0.61 in ten uniform ranges of normalized velocity magnitudes $v_{0}=|\mathbf{v}| / \max |\mathbf{v}|$.

\begin{tabular}{|c|c|c|c|c|c|c|}
\hline Normalized Velocity Magnitude & \multicolumn{2}{|l|}{$n_{e}=0.32$} & \multicolumn{2}{|l|}{$n_{e}=0.50$} & \multicolumn{2}{|l|}{$n_{e}=0.61$} \\
\hline$v_{0}=|\mathbf{v}| / \max |\mathbf{v}|$ & Flux $\%$ & Area\% & Flux $\%$ & Area\% & Flux $\%$ & Area\% \\
\hline $0.00 \leq v_{0} \leq 0.10$ & 14.61 & 60.16 & 4.87 & 27.60 & 3.19 & 18.67 \\
\hline $0.10<v_{0} \leq 0.20$ & 16.39 & 15.29 & 10.10 & 18.31 & 7.55 & 15.21 \\
\hline $0.20<v_{0} \leq 0.30$ & 17.07 & 9.45 & 14.10 & 15.16 & 13.74 & 16.61 \\
\hline $0.30<v_{0} \leq 0.40$ & 15.47 & 6.01 & 17.08 & 13.05 & 19.46 & 16.82 \\
\hline $0.40<v_{0} \leq 0.50$ & 14.81 & 4.42 & 17.55 & 10.47 & 24.11 & 16.28 \\
\hline $0.50<v_{0} \leq 0.60$ & 9.76 & 2.43 & 15.50 & 7.55 & 18.97 & 10.56 \\
\hline $0.60<v_{0} \leq 0.70$ & 5.69 & 1.19 & 10.96 & 4.50 & 9.02 & 4.28 \\
\hline $0.70<v_{0} \leq 0.80$ & 3.46 & 0.63 & 6.21 & 2.23 & 3.09 & 1.26 \\
\hline $0.80<v_{0} \leq 0.90$ & 2.19 & 0.35 & 2.60 & 0.82 & 0.73 & 0.27 \\
\hline $0.90<v_{0} \leq 1.00$ & 0.35 & 0.05 & 0.99 & 0.28 & 0.12 & 0.04 \\
\hline
\end{tabular}

curs. Principal pathways are also observed in Fig. 2 at other values of expected porosity.

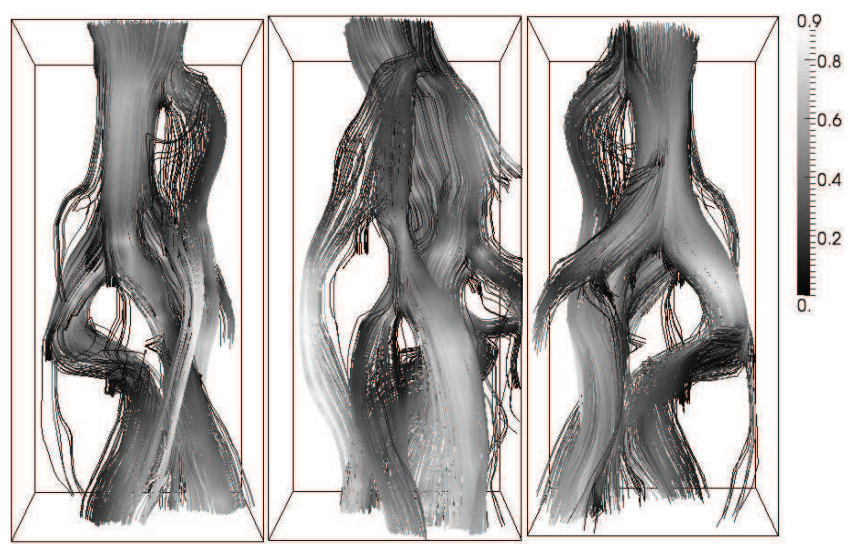

FIG. 8: Particle trajectories shaded according to their normalized velocity magnitude in an observation domain onesixteenth the size of the entire pore space realization. The second and third images are the same trajectories rotated about the vertical axis 90 degrees and 180 degrees. The particle are inserted within a disk centered atop the domain with a radius $1 / 5$ the domain width and the direction of flow is from top to bottom There are distinct regions of high velocity and low velocity along side one another in different pores.

\section{DISCUSSION}

The observed fluid flow across the thirty-four isotropic virtual pore space realizations is diverse (Fig. 2), and the flow within each porous medium is heterogeneous (Table III). At lower values of porosity, there are particles with short, fast, and straight trajectories, and others with long, slow, and twisted paths. Still other particles have various combinations of these attributes (Figs. 4, 5, and $6 \mathrm{a}-\mathrm{c})$. The number of dead-end pores in these porous media is substantial and results in a high degree of heterogeneity in the flow (Table III).

At higher values of porosity, the flow is fairly homogeneous (Table III). A majority of the particle trajectories have similar lengths, first passage times, and tortuosities. This uniformity results from smaller amounts of solid matrix in the porous media which yields decreased resistance to the fluid (Fig. 1). Yet, the long tails observed in first passage time distributions (Fig. 5) and trajectory length histograms (Fig. 6 d-f) reveal diversity in the seemingly uniform flow field.

The influence of porosity on the degree of nonuniformity in the fluid flow is manifested in the observed particle trajectory attributes of tortuosity, trajectory length, and first passage time. Low values of tortuosity result from straighter trajectories, and a decrease in tortuosity with increasing porosity was observed (Fig. 4). On the other hand, a nonlinear increase in mean tortuosity occurs with decreasing porosity. The proposed model (7) to predict mean tortuosity as a function of porosity is logarithmic and captures this nonlinearity. Moreover, the first passage time distributions (FPTD) exhibit a decrease in particle travel time at higher porosities, an indication of higher fluid velocities, and the sharpening of the FPTD at high porosities shows that the dissimilarity of travel times decreases, suggesting that the flow field is more uniform (Fig. 5).

The different shapes of the trajectory length distributions at various porosities are further evidence of porosity's influence on the fluid flow. At larger values of porosity the histograms gather around a mean value and exhibit a slight tail (Fig. 6 d-e). At the lowest value of expected porosity shown, distinct spikes appear at $\sim 3.10 \cdot 10^{-2} \mathrm{~m}$ and $\sim 3.40 \cdot 10^{-2} \mathrm{~m}$ (Fig. 6 a). As the porosity increases the variances decrease, the distributions are skewed to the right, and the maximum length of the particle trajectories decreases, as does the minimum (Table II). At low values of porosity, the kurtosis values are less than three, which indicates the distributions are less prone to have outliers than a normal distribution [54]. Conversely, at higher values of porosity the values of kurtosis are greater than three, which indicates that distributions of trajectory lengths are more prone to have outliers.

Additionally, the calculated value of mean tortuosity depends nonlinearly upon the vertical extent of the obser- 
vation domain and this dependence decays exponentially with increasing extent (Section III B). If the observation domain is not sufficiently long, the computed value of mean tortuosity underestimates the value obtained using the entire domain, consistent with [16]. As the extent of the observation domain increases, fluctuations in the computed values diminish and a representative elementary volume for tortuosity is observed. At higher values of porosity, the computed tortuosity converges to the limiting value of tortuosity at lower observational domain extents (Fig. 3). At lower porosities, the dependence of computed tortuosity on the extent of the observation domain is more significant. A similar dependence was found between the coefficient of variation of particle trajectory lengths and first passage times with the extent of the observational domain.

The bundling of trajectories is demonstrated by the grouping of particle exit locations in regions of high velocity and suggests that particles are drawn into principal pathways of flow. The pores traversed by these principal pathways are well connected, relatively straight, and the fluid within them moves quickly. If a fluid parti- cle enters such a principal pathway its tortuosity will be lower, trajectory length smaller, and first passage time shorter than a particles that does not. Determining the particular geometric features and topological structures of a medium that influence the formation and location of principal pathways is an open question.

\section{ACKNOWLEDGEMENTS}

We want to thank Alberto Guadagnini and Monica Riva for several discussions. J.D.H. acknowledges the support of the Graduate Visitor Program, a part of the Advanced Study Program at the National Center for Atmospheric Research. P.K.S. acknowledges partial support by the DOE awards \# DE-FG02-08ER64535 and \# DE-SC0006748 and the NSF grant OCI-0904599 while conducting this work. The National Center for Atmospheric Research is sponsored by the National Science Foundation.
[1] D. Klotz, K. P. Seiler, H. Moser, and F. Neumaier, J. Hydrol. 45, 169 (1980).

[2] B. Berkowitz, H. Scher, and S. E. Silliman, Water Resour. Res. 36, 149 (2000).

[3] P. C. Carman, J. Agric. Sci 29, 262 (1939).

[4] P. C. Carman, Flow of gases through porous media (Butterworths Scientific Publications London, 1956).

[5] A. Koponen, M. Kataja, and J. Timonen, Phys. Rev. E 54, 406 (1996).

[6] X. Shan and G. Doolen, Phys. Rev. E 54, 3614 (1996).

[7] A. Koponen, M. Kataja, and J. Timonen, Phys. Rev. E 56, 3319 (1997).

[8] R. S. Maier, D. M. Kroll, R. S. Bernard, S. E. Howington, J. F. Peters, and H. T. Davis, Phys. of Fluids 12, 2065 (2000).

[9] S. Succi, The lattice Boltzmann equation for fluid flow and beyond (Oxford Scientific publications, 2001).

[10] Z. Guo and T. S. Zhao, Phys, Rev. E 66, 036304 (2002).

[11] A. M. Tartakovsky and P. Meakin, Phys. Rev. E 72, 026301 (2005).

[12] A. M. Tartakovsky and P. Meakin, J. Comput. Phys. 207, 610 (2005).

[13] R. G. Erdmann, Image-based numerical simulation of Stokes flow in porous media (Ph.D. dissertation, Univ. Arizona, 2006).

[14] W. Fourie, R. Said, P. Young, and D. L. Barnes, in Proceedings of the Boston COMSOL Conference (2007).

[15] A. M. Tartakovsky, P. Meakin, T. D. Scheibe, and R. M. Eichler West, J. Comput. Phys. 222, 654 (2007).

[16] M. Matyka, A. Khalili, and Z. Koza, Phys. Rev. E 78, 026306 (2008).

[17] A. M. Tartakovsky and S. P. Neuman, Geophys. Res. Lett. 35, L21401 (2008).

[18] P. K. Smolarkiewicz and C. L. Winter, J. Comput. Phys. 229, 3121 (2010).
[19] Y. Zaretskiy, S. Geiger, K. Sorbie, and M. Förster, Adv. Water Res. 33, 1508 (2010).

[20] K. S. Alexander and S. A. Molchanov, J. Stat. Phys. 77, 627 (1994).

[21] K. S. Alexander, Ann. Probab. pp. 87-104 (1995).

[22] B. Kamgar-Parsi, B. Kamgar-Parsi, and M. Brosh, J. Stat. Comput. Sim 52, 399 (1995).

[23] J. M. Prusa, P. K. Smolarkiewicz, and A. A. Wyszogrodzki, Comput. Fluids 37, 1193 (2008).

[24] N. P. Wedi and P. K. Smolarkiewicz, J. Atmos. Sci. 63, 3226 (2006).

[25] P. K. Smolarkiewicz, R. Sharman, J. Weil, S. G. Perry, D. Heist, and G. Bowker, J. Comput. Phys. 227, 633 (2007).

[26] M. L. Waite and P. K. Smolarkiewicz, J. Fluid Mech. 606, 239 (2008).

[27] W. W. Grabowski and P. K. Smolarkiewicz, Mon. Weather Rev. 130, 939 (2002).

[28] B. J. Abiodun, J. M. Prusa, and W. J. Gutowski, Clim. Dynam. 31, 795 (2008).

[29] B. J. Abiodun, W. J. Gutowski, and J. M. Prusa, Clim. Dynam. 31, 811 (2008).

[30] P. Ortiz and P. K. Smolarkiewicz, Phys. Rev. E 79, 041307 (2009).

[31] M. Ghizaru, P. Charbonneau, and P. K. Smolarkiewicz, Astrophys. J. Lett. 715, L133 (2010).

[32] C. S. Peskin, J. Comput. Phys. 10, 252 (1972).

[33] R. Mittal and G. Iaccarino, Annu. Rev. Fluid Mech. 37, 239 (2005).

[34] J. H. Cushman and T. R. Ginn, Trans. Porous Media 13, 123 (1993).

[35] J. H. Cushman and M. Moroni, Phys. Fluids 13, 75 (2001).

[36] D. Goldstein, R. Handler, and L. Sirovich, J. Comput. Phys. 105, 354 (1993). 
[37] P. K. Smolarkiewicz and L. G. Margolin, J. Comput. Phys. 140, 459 (1998).

[38] P. K. Smolarkiewicz and J. M. Prusa, Turbulent flow computation (Springer, 2002), vol. 66, pp. 279-312.

[39] P. K. Smolarkiewicz and J. Szmelter, J. Comput. Phys. 228, 33 (2009).

[40] P. K. Smolarkiewicz, V. Grubišić, and L. G. Margolin, Monthly Weather Rev. 125, 647 (1997).

[41] P. K. Smolarkiewicz, C. Temperton, S. J. Thomas, and A. A. Wyszogrodzki, in ECMWF Seminar Series on Recent Developments in Numerical Methods for Atmospheric and Ocean Modelling (2004), pp. 6-10.

[42] J. D. Hyman, P. K. Smolarkiewicz, and C. L. Winter, Water Resourc. Res. (Submitted) (2012).

[43] J. Bear, Dynamics of fluids in porous media (Dover publications, 1988).

[44] A. Duda, Z. Koza, and M. Matyka, Phys. Rev. E 84, 036319 (2011).
[45] P. Xu and B. Yu, Adv. in Water Resour. 31, 74 (2008).

[46] A. H. Squillacote and J. Ahrens, The Paraview Guide (Kitware, 2006).

[47] S. Wolfram, The mathematica book (Cambridge university press, 1999).

[48] P. B. Lorenz, Nature 189, 386 (1961).

[49] H. L. Weissberg, J. of Appl. Phys. 34, 2636 (1963).

[50] J. Comiti and M. Renaud, Chem. Eng. Sci. 44, 1539 (1989).

[51] H. S. Salem and G. V. Chilingarian, Energy Sources 22, 207 (2000).

[52] L. Shen and Z. Chen, Chem. Eng. Sci. 62, 3748 (2007).

[53] L. Pisani, Trans. Porous Med. 88, 193 (2011).

[54] A. M. Mood, F. A. Graybill, and D. C. Boes, Introduction into the theory of statistics (McGraw-Hill International Book Company, 1963). 